\title{
Application of Multivariate Image Analysis to Thermal Images for on-line Monitoring of the Freeze-Drying Process
}

\section{Colucci, D. ${ }^{\text {a,b* }}$, Prats-Montalbán, J. M. ${ }^{\text {a }}$ Fissore, D. ${ }^{\text {b }}$ Ferrer, A. ${ }^{\text {a }}$}

${ }^{a}$ Multivariate Statistical Engineering Group, Department of Applied Statistics, Operations Research and Quality. Universitat Politècnica de València. Camino Vera s/n, 46022. València, Spain.

bDipartimento di Scienza Applicata e Tecnologia, Politecnico di Torino, Corso Duca degli Abruzzi 24, 10129 Torino, Italy.

*E-mail of the corresponding author: domenico.colucci@polito.it

\begin{abstract}
A new Process Analytical Technology (PAT) has been developed and tested for on-line process monitoring of a vacuum freeze-drying process. The sensor uses an infrared camera to obtain thermal images of the ongoing process and multivariate image analysis (MIA) to extract the information after automatic detection and segmentation of the region corresponding to the product in every vial. A reference model was built, using the information of six batches, and different kind of anomalous events, involving either single vials in the batch or the whole batch, were simulated to test the capacity of the MIA-based monitoring system to promptly identify them.
\end{abstract}

Keywords: freeze-drying; process monitoring; infrared image; multivariate image analysis. 
Application of Multivariate Image Analysis to Thermal Images for on-line Monitoring of the Freeze-Drying Process

\section{Introduction}

Vacuum freeze drying (VFD) is a highly attractive process for the water removal in thermal sensitive products, mainly pharmaceutical ones, since water is removed at low temperature by sublimation. Monitoring of critical quality attributes of the product, e.g. its temperature, is required to guarantee that the desired characteristics are obtained in the final product. Besides, it is required that the monitoring system does not interfere with product dynamics.

In the past, many approaches to this problem, based on the measurement of product temperature, sublimation rate, heat flux to the product, among the others, were proposed and tested [1], in particular at lab-scale. The measurement of the temperature of the product, possibly in a well-defined position (e.g. the bottom of the vial), was studied in detail and successfully applied for process monitoring and control [2]. The main drawback, up to this moment, of this approach is that the temperature measurement has to be performed using a thermocouple stuck into the product and this does not guarantee neither the sterility requirements nor that the sensor is not interfering with the ongoing process.

In this work we used an infrared camera, instead of a thermocouple, for temperature measurement. Differently from the system proposed in the literature, we placed the camera inside the chamber, thus being able to monitor the vials in several positions, and not only on the top shelf of the freeze-dryer [3], thus extending the monitoring capacity of the system. Unfortunately, thermal images include a lot of useless (i.e. everything that is outside the vial) information and also the one directly related with the process is highly noisy, redundant and correlated. The first problem is a matter of gray-scale image segmentation, and traditional image analysis, as well as computer vision, supply a lot of tools for working out this kind of matters; the second is a frequent problem when dealing with real industrial data, and latent based multivariate statistical techniques can easily deal with this kind of problems.

The idea underlying the development of a latent variable multivariate monitoring system is that only a few underlying events are driving the process, and all the measurements we obtain are just a different sight on this underlying driving force. Multivariate statistical methods allow us to obtain a model of the process by projecting the information into a low dimensional space defined by latent variables and, in this reduced space, we can build control charts able to detect any deviation from the normal operating conditions [4]. Principal component analysis (PCA) has been widely studied and applied for this purpose being also able to successfully deal with the highly auto-correlated and cross-correlated data typical of batch processes [5] such as VFD intrinsically is. Multivariate control charts for batch process monitoring have been proposed by Nomikos and MacGregor [6]. 


\section{Materials and Methods}

\subsection{Experimental}

Drying experiments were carried out using a lab scale equipment LyoBeta $25^{\mathrm{TM}}$ freeze-dryer (Telstar, Spain). In all tests ten vials (ISO 8362-1 8R) were placed at $30 \mathrm{~cm}$ from the camera, and a new image was acquired every five minutes. Each vial in the batches has been assigned with a number referring to the position in the shelf as Figure 1 shows.

The normal operating conditions (NOC) set was obtained processing a solution of $10 \%$ b.w. sucrose (Sigma Aldrich, 99.5\%), $5 \mathrm{ml}$ per vial, at $-20^{\circ} \mathrm{C}$ and $20 \mathrm{~Pa}$. Six batches were processed in the same operating conditions, thus obtaining 60 observations, corresponding to the 10 vials monitored in the 6 tests. The detection ability of the system was evaluated in four additional batches (i.e. observations from 61 to 100). In batch 7, after 5 hours of drying, chamber pressure was raised to $50 \mathrm{~Pa}$; in batch 8 the shelf temperature was set to $-10^{\circ} \mathrm{C}$ while in batch 10 a solution $5 \%$ b.w. of sucrose was used. Batch 9 aimed to prove the ability of the model to detect faults affecting the single vials and, while shelf temperature and chamber pressure were set to the NOC values, only four vials (corresponding to observations 81, 88, 89 and 90 ) were filled with the $10 \%$ solution. A piece of glass was inserted into two vials, one was filled with pure water, one with the same $5 \%$ b.w. solution used for batch 10 and the remaining two with respectively 2.5 and $7.5 \mathrm{ml}$ of solution.

Due to the vibrations of the equipment, during batch three, vial 7 felt down and was considered as a fault, while the remaining nine vials were considered successfully dried.

\subsection{Image segmentation and data acquisition}

The thermal images are $256 \times 320$ pixels. The camera is equipped with a $63^{\circ} \times 50^{\circ}$ lens which leads to a slight optical distortion, known as barrel effect. This second order deviation from the ideal rectilinear projection can be compensated remapping the pixels according to the following equation:

$$
r_{\text {new }}=r_{\text {old }}+f \cdot r_{\text {old }}^{2}
$$

where $r$ is the distance from the center of the image of a generical pixel and $f$ a correction factor (negative in this kind of optical aberration) depending on the distance between the camera and the object [7]. In all our tests the same distance was used this factor is approximately constant and equal to -1.5 .

After optical correction, the Hough transform [8] was used to detect the position of the vials in the images, as shown in Figure 1. Being known the diameter of the vials bottom and the length of the line detected by the Hough transform we were able to infer the width of a single pixel and thus, as we know also the height of the vial, the height of the mask. 
Application of Multivariate Image Analysis to Thermal Images for on-line Monitoring of the Freeze-Drying Process

The whole portion of the image corresponding to the product into every vial was segmented and, to study the evolution over time of the temperature distribution, mean, standard deviation (std), skewness and kurtosis of the temperature in this region were measured. The results were collected into a three-dimensional data structure $\underline{\mathbf{X}}(I \times J \times K)$ where $I$ is the number of observation, being each vial intended as a single observation, $J$ is the number of variables measured, and $K$ is the number of time instants.

\subsection{Batch process monitoring}

The data structure was batch-wise unfolded obtaining a matrix $\mathbf{X}(I \times J K)$ [5]. After mean centering and scaling $\mathbf{X}$, a PCA model with $A$ principal component was built using the available observations, obtained in batch 1 to 6 .

$$
\mathrm{X}=\mathrm{T} \cdot \mathrm{P}^{\prime}+\mathrm{E}
$$

where $\mathbf{T}$ is the $I \times A$ score matrix, $\mathbf{P}$ is the $A \times J K$ loading matrix and $\mathbf{E}$ is the $I \times J K$ residual matrix. The number of principal components that maximizes the classification skills of the model was determined as that who maximizes the following function:

$$
f_{A}=(1-\alpha) \cdot(1-\beta)
$$

where $\alpha$ is the overall type I error rate (i.e. false positive rate) and $\beta$ is the overall type II error rate (i.e. false negative rate). Once the latent variable sub-space is known, unusual behaviors can be detected using two multivariate control charts: Hotelling $\mathrm{T}^{2}\left(T^{2}\right)$ and the squared prediction error (SPE) defined by equations 4 and 5 for each observation:

$$
T^{2}=\sum_{a=1}^{A} \frac{t_{a}^{2}}{\lambda_{a}}
$$

$$
S P E=\sum_{c=1}^{K J} e_{c}^{2}
$$

where $t_{a}$ is the $a$-th score and $e_{c}$ is the error obtained after predicting the measurement of variable $c$ for a certain observation. Upper control limits (UCL) for these charts were computed both empirically, that is taking the percentile of the actual values measured for the same NOC observations, and using their theoretical statistical distributions [6]. Once the number of principal components has been fixed, the possible outliers that would contribute to raise the control limits have been detected and purged. Due to the reduced number of batches available, a one-batch-out cross-validation approach was used: each batch is in turn removed from the NOC data set used to build the PCA, all the observations are projected, and those observations that prove to be outliers are removed.

The main issue when dealing with on-line multivariate SPC is the imputation of the missing information [6], a good approach being regression on the available NOC data used to build the PCA model [9]. In this work the Trimmed Score Regression (TSR) method has been used [10]. In on-line monitoring the SPE is computed only on the information measured at instant $k$, and for this reason is called instant SPE (SPEI):

$21^{\text {ST }}$ INTERNATIONAL DRYING SYMPOSIUM EDITORIAL UnIVERSITAT POLITÈCNICA DE VALÈNCIA 
Colucci, D.; Prats-Montalbán; J. M. ; Fissore, D.; Ferrer, A.

$$
S P E I_{c}=\sum_{c=1+(k-1) J}^{K J} e_{c}^{2}
$$

Nomikos and MacGregor proposed to use the errors on a moving window of five instant measurements to compute the UCL limits for this statistic [6]. The percentage of time instants that SPEI overtake the UCL in in-control batches, also called Overall type I (OTI) risk, should be lower than the imposed significance level (ISL $=0.5 \%$ ) and for this reason the UCL have been manually tuned [11]. $N f$ is the number of instantaneous faults for the overall trial set.

$$
O T I=100 \cdot \frac{N f}{I \cdot K} \%
$$

\section{Results}

Figure 2 reports an example of the 10 trajectories, one for each vial, described by the four variables measured during the drying process in one of the reference batch. Average temperature shows an asymptotic behavior up to the thermal equilibrium. The standard deviation (std), after a sudden decrease, grows up until a maximum is reached at almost $9 \mathrm{~h}$; then, it slowly decreases again until reaching an almost constant value at $36 \mathrm{~h}$. Both skewness and kurtosis show a maximum, followed by a local minimum around 9 hours. An almost constant value is kept from $36 \mathrm{~h}$ to the end. The local maxima (or minima) seems to correspond to the first slope change of the Pirani/Baratron pressure ratio. The constant values at the end indicates that the thermal equilibrium has been reached and the primary drying is over. Significant differences in these thermal trajectories may reveal an abnormal heat transfer, that is an anomalous drying kinetic and a lower product quality.

From the analysis of the scree plot and the cumulative variances plot (not reported) we decided to evaluate $f_{A}$ for a number of components going from 5 to 13 , that is from $83.9 \%$ to $95.4 \%$ of the whole variance. Figure 3 shows that $f_{A}$ clearly shows a maximum when 10 PC are used (93.6\% variance explained). Observation 21 appeared as the only outlier during the one-batch-out cross-validation step, and for this reason has been removed from the final data set. The UCL for $T^{2}$, depending only on the number of PC extracted and the number of observations, are constant but there is a remarkable difference between the theoretical and the empirical value; being the former (20.9) always lower than the latter (36.3). On the other hand, the UCL for SPEI computed with the theoretical distribution and the one obtained taking the percentile of $99.5 \%$ are always very similar. After tuning the control limits, the obtained OTIs were $0.496 \%$ for SPEI and zero for $T^{2}$.

The classification performance of this monitoring system has been evaluated by projecting all the batches (one hundred vials/observations) on the obtained model. Tuned empirical limits performed slightly better than the theoretical ones and have been used. Figure 4 shows an example of the resulting control chart for three observations: number 5, a vial of the NOC set, always below the UCL; number 21 that was removed as an outlier and has been detected as a fault by the SPEI control chart only; and number 75 dried at a higher shelf temperature 
Application of Multivariate Image Analysis to Thermal Images for on-line Monitoring of the Freeze-Drying Process

and lies over the control limits of both statistics almost all the time. The control charts for SPEI detected 10 false positives observations in the trial set $(6,9,10,15,16,19,25,31,40$, 50). Observations 21 and 27 (the one that felt down in batch 3) have been detected as faults as well as all the observations of the anomalous batches 7, 8 and 10. In batch 9 six vials were tampered and all of them have been correctly discriminated, while all the remaining good vials resulted into false positives. The $T^{2}$ control chart reported thirteen false negatives. Only two of the four vials of batch 9 dried with the original 10\% sucrose solution (observations 81 and 90) have been correctly found to be successful drying tests. Observations 88 and 89 have been highlighted as faults.

Looking at the observations that appeared as false positives in SPEI (they are 6, 9, 10, 15, $16,19,25,31,40,50$ ) we can notice a certain periodicity in the results. Position 1 and 10 in every batch corresponds to the external vials, directly radiated by the chamber walls. Observation 21, as well as 81 and 90, appear to support this hypothesis. In the first three batches a thermocouple was located inside the vials in position 5 and 6, see Figure 2. This slight difference into the data structure of observation 6, 15, 16 and 25 might be due to the influence of the thermocouple on the drying kinetics.

These observations overtake the control limits on a limited number of time instants. If we accept these spurious errors as part of the unavoidable statistical error rate, that is, we consider the phenomena responsible for these instantaneous faults cannot jeopardize the quality of the resulting product, the fault detection performance could be further optimized. This new relaxation of the control limits was achieved by considering faults only the observations that cross the control limits in more than $5 \%$ of the time instants. In this way all the false positives in SPEI where properly classified as successful tests except observations 81 and 90. No further improvement was possible for Hotelling $T^{2}$ control charts since lowering the UCL to include the false negatives raises new false positives. This lower detection ability of $T^{2}$ control charts, when dealing with data from batch processes, has already been reported in the literature and is basically due to the strong auto-correlation in the data [11].

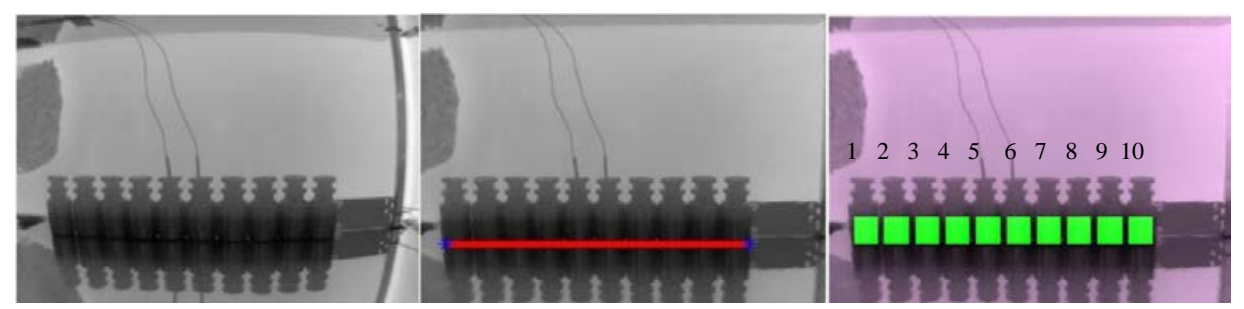

Fig. 1: Image preprocessing and segmentation. 
Colucci, D.; Prats-Montalbán; J. M. ; Fissore, D.; Ferrer, A.
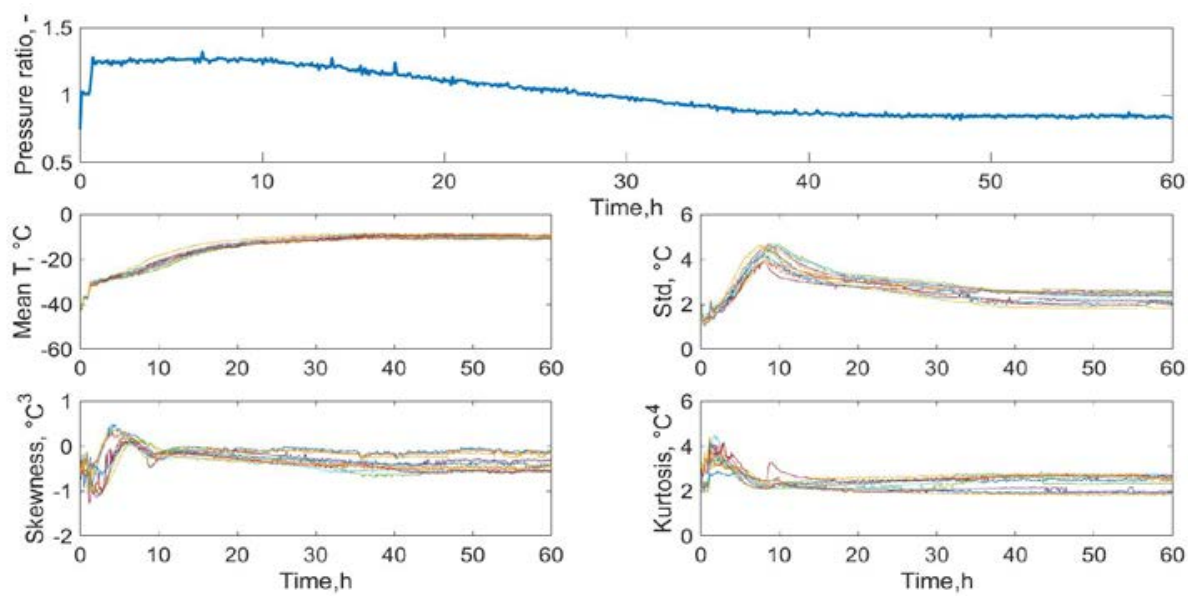

Fig. 2: Pirani Baratron ratio and temperature trajectories

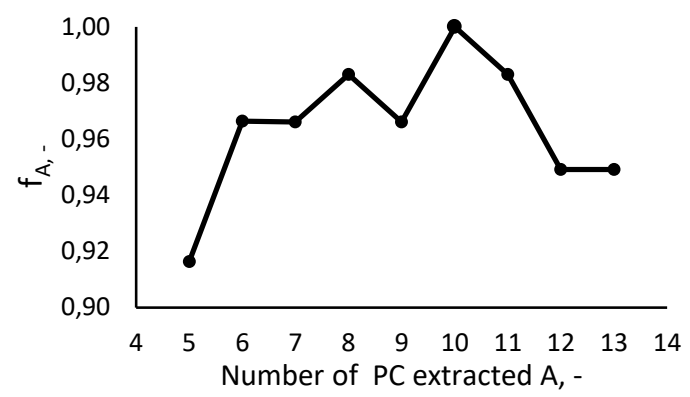

Fig. 3: Optimal number of Principal Components
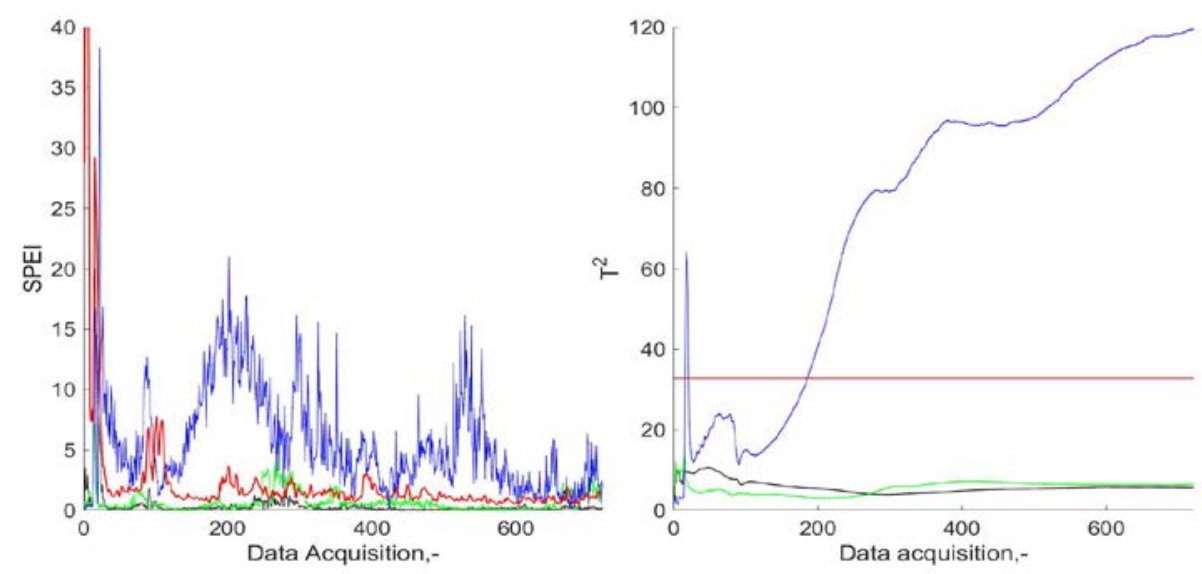

Fig. 4: Control charts for observation 5 (black line) and 21(signaling only in SPEI, green line) and 75 (signaling in SPEI an $T^{2}$, blue line). Red line tuned empirical UCLs. 
Application of Multivariate Image Analysis to Thermal Images for on-line Monitoring of the Freeze-Drying Process

\section{Conclusions}

In this work MIA techniques have been applied to thermal images of the VFD process and a PAT for real time monitoring have been developed and successfully tested. The sensor, using an infrared camera, is completely non-invasive and have no side-effects on the process kinetics. This technology proved to be quite sensitive being able to discriminate the slight effect on the product of either a thermocouple inserted into some vials or chamber walls radiation. Future works will aim to prove the possibility to use this PAT and the infrared imaging technology for process control and optimization purposes.

\section{References}

[1] Fissore, D.; Pisano, R.; Barresi, A.A. Process analytical technology for monitoring pharmaceuticals freeze-drying - A comprehensive review. Drying Technology, In press (DOI: 10.1080/07373937.2018.1440590).

[2] Fissore, D.; Pisano, R.; Barresi, A.A. On the use of temperature measurement to monitor a freeze-drying process for pharmaceuticals. In Proceedings of I2MTC - 2017 IEEE International Instrumentation and Measurement Technology Conference, May 22-25, Torino, Italy. 1276-1281

[3] Emteborg, H.; Zeleny, R.; Charoud-Got, J.; Martos, G.; Luddeke, J.; Schellin, H.; Teipel, K. Infrared thermography for monitoring of Freeze-Drying processes: instrumental developments and preliminary results. Journal of Pharmaceutical Sciences 2014, 103, 2088-2097.

[4] Kourti, T; MacGregor, J.M. Multivariate SPC methods for process and product monitoring. Journal of Quality Technology 1996, 28(4), 409-428.

[5] Kourti, T. Multivariate dynamic data modeling for analysis and statistical process control of batch processes, start-ups and grade transitions. Journal of Chemometrics 2003, 17, 93-109.

[6] Nomikos, P.; MacGregor, J.M. Multivariate SPC charts for monitoring batch processes. Technometrics 1995, 37, 41-59.

[7] De Villiers, J.P.; Leuschner, F.W.; Geldenhuys, R. Centi-pixel accurate real-time inverse distortion correction. In Proceedings of SPIE, 2008, 7266, 11-1-11-8.

[8] Gonzalez, R.C.; Woods, R.E.; Eddins, S.L. Digital image processing using Matlab. Pearson education, Inc.: Upper Saddle River, New Jersey, 2004.

[9] Arteaga, F.; Ferrer, A. Framework for regression-based missing data imputation methods in on-line MSPC. Journal of Chemometrics 2005, 19, 439-447.

[10] Arteaga, F.; Ferrer, A. Dealing with missing data in MSPC: several methods, different interpretations, some examples. Journal of Chemometrics 2002, 16, 408-418.

[11] Camacho, J.; Picó, J.; Ferrer, A. Multi-phase analysis framework for handling batch process data. Journal of Chemometrics 2008, 22, 632-643. 